Pacific Journal of Mathematics

SINGULARITIES OF A HARMONIC FUNCTION OF THRE 


\title{
SINGULARITIES OF A HARMONIC FUNCTION OF THREE VARIABLES GIVEN BY ITS SERIES DEVELOPMENT
}

\author{
Alvin M. White
}

Introduction. The connection between complex function theory and the theory of partial differential equations as developed by Bergman [4] allows one to translate many properties of functions of complex variables into corresponding properties of solutions of partial differential equations.

Integral operators introduced and studied by Bergman transform analytic functions into solutions of elliptic partial differential equations with analytic coefficients. Under the Bergman integral operator, there is a one-to-one correspondence between harmonic functions of three real variables defined in a neighborhood of the origin and analytic functions of two complex variables. This is a correspondence in a sufficiently small neighborhood. By applying the method of continuation one passes to a correspondence in the large. In this way some singularities of analytic functions are translated into singularties of harmonic functions in the global sense. Investigations in this direction were conducted by Bergman [2], [3], [5], Kreyszig [9], [10], Mitchell [12], Gilbert [7] and the author [14].

The present paper is concerned with further relations between singularities of harmonic functions and the analytic functions with which they are associated. By means of the operator, criteria for a harmonic function to have certain singularities are found by considering subsequences of coefficients of a series development of the harmonic function.

The Bergman operator is introduced in $\S 1$. In $\S 2$ some theorems relating subsequences of coefficients of a series development and possible singularity curves are proved. It is proved in $\S 3$ that the harmonic function represented by a Bergman operator of a certain general form also satisfies a system of ordinary linear differential equations. A special case of this result is discussed in $\S 4$.

1. The Whittaker-Bergman operator for harmonic functions. The operator

$$
\widetilde{H}(x, y, z)=\frac{1}{2 \pi i} \int_{0}^{2 \pi} f\left(u, e^{i t}\right) d t
$$

transforms an analytic function $f\left(u, e^{i t}\right)$ of $e^{i t}$ and

Received May 16, 1962. Sponsored by the Mathematics Research Center, U.S. Army, Madison, Wisconsin under Contract No. DA-11-022-ORD-2059. The author wishes to thank Professor V. Maric' for his valuable help. 


$$
u=x+i(y \cos t+z \sin t)
$$

into a harmonic function of three real variables $x, y, z$ [4].

Setting $e^{i t}=\zeta$ and

$$
X=x, Z=\frac{1}{2}(z+i y), Z^{*}=-\frac{1}{2}(z-i y)
$$

we have

$$
u=X+Z \zeta+Z^{*} \zeta^{-1}
$$

and (1.1) becomes

$$
H\left(X, Z, Z^{*}\right)=\frac{1}{2 \pi i} \int_{|\zeta|=1} f(u, \zeta) \frac{d \zeta}{\zeta} .
$$

The generated function $H\left(X, Z, Z^{*}\right)$ is complex valued. Taking the real part, we obtain a real harmonic function [3], [5].

2. Some relations between subsequences of coefficients of a series development of a harmonic function and its singularities. Bergman [2] has shown that every (complex) harmonic function of three variables defined in a neighborhood of the origin can be represented in a sufficiently small neighborhood of the origin in a series of the form

$$
\sum_{m=0}^{\infty} \sum_{k=-m}^{m} \frac{a_{m+k, m}}{2 \pi i} \int_{|\zeta|=1} u^{m} \zeta^{k} \frac{d \zeta}{\zeta},
$$

where

$$
\frac{1}{2 \pi i} \int_{|\zeta|=1} u^{m} \zeta^{k} \frac{d \zeta}{\zeta}=\frac{i^{m} k !}{(k+m) !} r^{m} P_{k}^{m}(\cos \theta) e^{i m \varphi}
$$

$P_{k}^{m}(\cos \theta)$ are associated Legendre polynomials ([15], p. 392) and $r, \theta, \varnothing$ are spherical coordinates ([4], p. 42).

In a sufficiently small neighborhood of the origin the series (2.1) converges absolutely and uniformly. The coefficients $\left\{a_{m+k, m}\right\}$ form a triangular array

$$
\begin{array}{cccccccc}
\multicolumn{8}{c}{a_{00}} \\
& a_{01} & a_{11} & a_{21} & & & \\
& a_{02} & a_{12} & a_{22} & a_{32} & a_{42} & \\
a_{03} & a_{13} & a_{23} & a_{33} & a_{43} & a_{53} & a_{63}
\end{array}
$$

Information about the character and the location of the singularities of the harmonic function can be obtained from the coefficients (2.2) of the representation (2.1). 
For future reference, Hadamard's theorem on polar singularities will be stated [8], [6].

Let

$$
f(z)=\sum_{0}^{\infty} c_{n} z^{n}
$$

Define

$$
l_{p}=\varlimsup_{n=\infty}\left|\sqrt[n]{D_{n}^{(p)}}\right|
$$

where

$$
D_{n}^{(p)}=\left|\begin{array}{llll}
c_{n} & c_{n+1} & \cdots & c_{n+p} \\
c_{n+1} & c_{n+2} & \cdots & c_{n+p+1} \\
\cdots & \cdots & \cdots & \cdots \\
c_{n+p} & c_{n+p+1} & \cdots & c_{n+2 p}
\end{array}\right|
$$

Necessary and sufficient conditions that $f(z)=\sum c_{n} z^{n}$ should have at most $p$ poles and no other singularities on the circumference of its circle of convergence are that

$$
l_{i}=\frac{1}{r^{i+1}}, \quad i=0,1,2, \cdots, p-1 \quad \text { and } \quad l_{p}<\frac{1}{r^{p+1}} .
$$

It is then possible to determine a polynomial

$$
P(z)=1+a_{1} z+\cdots+a_{p} z^{p}
$$

such that the radius of $P(z) f(z)$ is $>r$ and the poles of $f(z)$ are the roots of $P(z)=0$.

By considering subseries of (2.1) summed over a single index it is possible to apply Hadamard's theorem and relate subsequences of coefficients of a series development to singularities of the harmonic function.

\section{THEOREM 2.1. Let}

$$
\begin{aligned}
H\left(X, Z, Z^{*}\right) & =\frac{1}{2 \pi i} \sum_{m=0}^{\infty} \sum_{k=-m}^{m} a_{m+k, m} \int_{|\zeta|=1} u^{m} \zeta^{k} \frac{d \zeta}{\zeta} \\
F_{k_{0}}\left(X, Z, Z^{*}\right) & =\frac{1}{2 \pi i} \sum_{m=0}^{\infty} a_{m+k_{0}, m} \int_{|\zeta|=1} u^{m} \zeta^{k_{0}} \frac{d \zeta}{\zeta} .
\end{aligned}
$$

With the coefficients $\left\{a_{m+k_{0}, m}\right\}$ associate the numbers

$$
l_{p}^{\left(k_{0}\right)}=\varlimsup_{m=\infty}\left|D_{m, p}^{\left(k_{0}\right)}\right|^{1 / m}, \quad \quad p=0,1,2, \cdots
$$

where 


$$
D_{m, p}^{\left(k_{0}\right)}=\left|\begin{array}{llll}
a_{m+k_{0}, m} & a_{m+k_{0}, m+1} & \cdots & a_{m+k_{0}+p, m+p} \\
a_{m+k_{0}+1, m+1} & a_{m+k_{0}+2, m+2} & \cdots & a_{m+k_{0}+p+1, m+p+1} \\
\cdots & \cdots & \cdots & \cdots \\
a_{m+k_{0}+p, m+p} & a_{m+k_{0}+p+1, m+p} & \cdots & a_{m+k_{0}+2 p, m+2 p}
\end{array}\right|
$$

If $l_{i}^{\left(k_{0}\right)}=1 / r^{i+1}, i=0,1,2, \cdots, p-1$, and $l_{p}^{\left(k_{0}\right)}<1 / r^{p+1}$ then it is possible to determine a polynomial $P_{k_{0}}(u)$ of degree $p$ see (2.5). If $P_{k_{0}}(u)=0$ has no multiple roots and $\left[H-F_{k_{0}}\right]$ is an entire harmonic function then (2.6) has $p$ branch line singularities [4] on the sphere $x^{2}+y^{2}+z^{2}=r^{2}$. The branch lines lie in the planes $x=x_{\nu}$, where $\left|x_{\nu}\right|>\left|x_{\nu+1}\right| ; x_{1}=r \cos \xi_{1}$ and $\xi_{\nu}$ can be determined from the coefficients $\left\{a_{m+k_{0}, m}\right\}$ see [3].

Proof. In a sufficiently small neighborhood (2.7) can be written as

$$
F_{k_{0}}\left(X, Z, Z^{*}\right)=\frac{1}{2 \pi i} \int_{|\zeta|=1} q_{k_{0}}(u) \zeta^{k_{0}-1} d \zeta
$$

where

$$
q_{k_{0}}(u)=\sum_{m=0}^{\infty} a_{m+k_{0}, m} u^{m} .
$$

The necessary and sufficient conditions that $q_{k_{0}}(u)$ should have at most $p$ poles and no other singularities on its circle of convergence are that

$$
l_{i}^{\left(k_{0}\right)}=\frac{1}{r^{i+1}}, \text { for } i=1,2, \cdots, p-1 \text { and } l_{p}^{\left(k_{0}\right)}<\frac{1}{r^{p+1}} \quad[6],[8] .
$$

Denote the poles on the circle of convergence by

$$
\alpha_{1}, \alpha_{2}, \cdots, \alpha_{p} \text {. }
$$

By hypothesis, the poles are simple. $q_{k_{0}}(u)$ can then be written in the form

$$
q_{k_{0}}(u)=\sum_{\nu=1}^{p} \frac{b_{\nu}}{\left(u-\alpha_{\nu}\right)}+Q(u)
$$

where $Q(u)$ is a power series with radius of convergence greater than $r$. If $q_{k_{0}}(u)$ has singularities only at $\alpha_{1}, \cdots, \alpha_{p}$, then $Q(u)$ is an entire function.

Carrying out the integration as in (2.7)' obtain

$$
\frac{1}{2 \pi i} \sum_{\nu=1}^{p} b_{\nu} \int_{|\zeta|=1} \frac{\zeta^{k_{0}-1} d \zeta}{u-\alpha_{\nu}}+\frac{1}{2 \pi i} \int_{|\zeta|=1} Q(u) \zeta^{k_{0}-1} d \zeta .
$$

When $k_{0}=0$ the first term (for fixed $\nu$ ) is 


$$
\frac{b_{\nu}}{2 \pi i} \int_{|\zeta|=1} \frac{d \zeta}{\left(u-\alpha_{\nu}\right) \zeta}=\frac{1}{\sqrt{\left(x-\alpha_{\nu}\right)^{2}+y^{2}+z^{2}}}
$$

where the square root must be chosen so that the quantity

$$
\frac{-\left(x-\alpha_{\nu}\right)+\sqrt{\left(x-\alpha_{\nu}\right)^{2}+y^{2}+z^{2}}}{(i y+z)}
$$

(one of the two zeros of the denominator of the integrand) lies within the unit circle. The integral is well-defined for those points where $x \neq\left(\operatorname{Re} \alpha_{\nu}\right)$, and for the plane $x=\left(\operatorname{Re} \alpha_{\nu}\right)$ those points which also satisfy $y^{2}+z^{2}<\left(\operatorname{Im} \alpha_{\nu}\right)^{2}$. The function (2.14) constitutes one branch of a two valued function which becomes infinite along the circle.

$$
x^{2}+y^{2}+z^{2}=r^{2}, \quad x=\left(\operatorname{Re} \alpha_{\nu}\right)=r \cos \xi_{\nu} .
$$

The circle is a "branch-line" analogous to a branch-point of multiple valued functions of a complex variable.

The poles (2.11) all lie on the same circle of radius $r$ and can be denoted as

$$
\alpha_{\nu}=r\left(\cos \xi_{\nu}+i \sin \xi_{\nu}\right) .
$$

For $k_{0}>0$, the branch lines are the same ([4], p. 47).

The value of $\left|\xi_{1}\right|$ such that $\left|\xi_{1}\right|<\left|\xi_{\nu}\right|, \nu \neq 1$, can be determined by applying the following theorem of Mandelbrojt [11]:

Without loss of generality, suppose the circle of convergence of $f(z)=\sum a_{n} z^{n}$ is unity. Put $d_{n}(h)=a_{0} h^{n}+C_{n}^{1} a_{1} h^{n-1}+\cdots a_{n},(h \geqq 0)$, and $C_{i}^{j}$ are the binomial coefficients.

$$
R(h)=\varlimsup_{n=\infty} \sqrt[n]{\left|d_{n}(h)\right|}, \quad[R(0)=R=1] .
$$

The function $R(h)$ possesses, for $h=0$, a derivative from the right

$$
R^{+\prime}(0)=\lim _{h=+0} \frac{R(h)-1}{h}
$$

and $\left|R^{+\prime}(0)\right| \leqq 1$.

Putting $R^{+\prime}(0)=\cos \varphi$, one of the points $e^{ \pm i \varphi}$ is the singular point of $f(z)$ which is on the circle of convergence nearest the point 1 .

$\left|\xi_{1}\right|$ can be found by setting

$$
\frac{1}{r} a_{m+k_{0}, m}=a_{n}
$$

and applying Mandelbrojt's theorem. To obtain the argument of $\left(\alpha_{\sigma}\right)$, that is, the value of $\left|\xi_{\sigma}\right|$, apply the same procedure to

$$
\prod_{\nu=1}^{\sigma-1}\left(u-\alpha_{\nu}\right) q_{k_{0}}(u) \text {. }
$$


A special case of Theorem 2.1 was proved by Bergman [3], [5].

The series (2.1) representing a harmonic function can be summed according to various arrangements. In Theorem 2.1 the distinguished subseries correspond to vertical columns in the array (2.2).

The branch lines corresponding to polar singularities of these distinguished subseries are circles with centers on the $x$-axis lying in planes normal to the $x$-axis.

Developing our considerations, it is of interest to introduce a new operator (suggested by Bergman)

$$
\begin{gathered}
\tilde{H}=\mathbf{W}(H)=\mathbf{W}\left(F^{*}\right)+\mathbf{W}\left(H-F^{*}\right), \\
F^{*}\left(X, Z, Z^{*}\right)=\frac{1}{2 \pi i} \sum_{n=0}^{\infty} a_{2 n, n} \int_{|\zeta|=1} u^{n} \zeta^{n} \frac{d \zeta}{\zeta},
\end{gathered}
$$

generating harmonic functions of such nature that to some properties of $\left\{a_{2 n, n}\right\}$ correspond singularities of $\widetilde{H}=\widetilde{H}\left(X, Z, Z^{*}\right)$ of a certain type.

One can obtain an operator $\mathbf{W}$ as follows: let $h(\tau)=\sum_{i=0} a_{2 n, n} \tau_{n}$ and let $\left\{a_{n}\right\}$ satisfy the Hadamard conditions insuring that $h(\tau)$ has finitely many poles on the circle of convergence as the only singularities in $|\tau|<\infty$. If in addition one requires that these poles are of the first order, then

$$
h(\tau)=\sum_{\mu=0}^{m} \frac{b_{\mu}}{\tau-\beta_{\mu}}+g(\tau),
$$

and $g(\tau)$ is an entire function. We define

$$
\mathbf{W}\left(F^{*}\right)=\frac{1}{2 \pi i} \int_{s}\left[\sum_{\mu=0}^{m} \frac{b_{\mu}}{u \zeta-\beta_{\mu}}+g(u \zeta)\right] \frac{d \zeta}{\zeta}
$$

where $s$ is no longer the unit circle, but a conveniently chosen closed curve in the $\zeta$-plane which contains the origin. Then the harmonic function $\widetilde{H}$ will possess singularities of a certain type. Notice that if $G$ is an entire harmonic function, then $\mathbf{W}(G)=G$. We shall discuss two applications of this idea from which the details of the procedure will be clearer.

If (2.1) is summed according to a sequence of coefficients which are parallel to the left or right leg of the triangular array (2.2), then the branch lines corresponding to polar singularities of these distinguished subseries will be circles which are tangent to the $x$-axis at the origin.

THEOREM 2.2. Let

$$
\begin{aligned}
& \tilde{H}=\mathbf{W}(H)=\mathbf{W}\left(F^{*}\right)+\mathbf{W}\left(H-F^{*}\right) \\
& H-F^{*}=\frac{1}{2 \pi i} \sum_{m=0}^{\infty} \sum_{k=-m}^{m-1} a_{m+k, m} \int_{|\zeta|=1} u^{m} \zeta^{k} \frac{d \zeta}{\zeta} .
\end{aligned}
$$


With the coefficients $\left\{a_{2 n, n}\right\}$ (which is the subsequence along the right leg of (2.2)) associate the numbers

$$
l_{p}^{*}=\varlimsup_{n=\infty}\left|D_{n}^{* p}\right|^{1 / n}
$$

where

$$
D_{n}^{* p}=\left|\begin{array}{llll}
a_{2 n, n} & a_{2 n+2, n+1} & \cdots & a_{2 n+2 p, n+p} \\
a_{2 n+2, n+1} & a_{2 n+4, n+2} & \cdots & a_{2 n+2 p+2, n+p+1} \\
\cdots & \cdots & \cdots & \cdots \\
a_{2 n+2 p, n+p} & a_{2 n+2 p+2, n+p+2} & \cdots & a_{2 n+4 p, n+2 p}
\end{array}\right| .
$$

If $l_{i}^{*}=1 / r^{i+1}, i=0,1,2, \cdots p-1$ and $l_{p}^{*}<1 / r^{p+1}$ then it is possible to determine a polynomial $P^{*}(w)$ of degree $p$ see (2.5). If $P^{*}(w)=0$ has no multiple roots, the above poles are the only singularities of $\sum a_{2 n, n} \tau^{n}$, and $\left[H-F^{*}\right]$ is an entire harmonic function then (2.22) has $p$ branch-line singularities. Each branch-line is along a circle in $x, y, z$ space which is tangent to the $x$-axis at the origin, the planes of the circles contain the $x$-axis, and the centers of the branch-line circles all lie on the circle

$$
x^{2}+y^{2}+z^{2}=r^{2}, \quad x=0 .
$$

REMARK. Using the consideration of the first part of this section, one can establish conditions for $\left\{a_{2 n, n}\right\}$ in order that $h(\tau)$ has distinct poles and that these poles are the only singularities of $h$.

Proof. From the definition of $\mathbf{W}$ and our assumptions it follows that

$$
\widetilde{H}=\frac{1}{2 \pi i} \int_{s}\left[\sum_{\mu=0}^{m} \frac{b_{\mu}}{u \zeta-\beta_{\mu}}+g(u \zeta)\right] \frac{d \zeta}{\zeta}+\left(H-F^{*}\right) \text {. }
$$

Since $H-F^{*}$ and $g(\tau)$ are entire functions, we get the above described singularities by carrying out the integration

$$
\frac{1}{2 \pi i} \sum_{\mu=1}^{p} b_{\mu} \int_{s} \frac{d \zeta}{\left(u \zeta-\beta_{\mu}\right)} \zeta
$$

For fixed $\mu$ this gives

$$
\begin{aligned}
& \frac{b_{\mu}}{2 \pi i} \int_{s} \frac{d \zeta}{\left(u \zeta-\beta_{\mu}\right) \zeta} \\
& \quad=\frac{b_{\mu}}{Z^{*}-\beta_{\mu}}+\frac{2 b_{\mu} Z}{\left(-x+\sqrt{x^{2}-4 Z\left(Z^{*}-\beta_{\mu}\right)}\right) \sqrt{x^{2}-4 Z\left(Z^{*}-\beta_{\mu}\right)}},
\end{aligned}
$$

where the curve $s$ is chosen so that besides $\zeta=0$ only one of the roots of $Z \zeta^{2}+X \zeta+\left(Z^{*}-\beta_{\mu}\right)=0$ lies within $s$ and where $\beta_{\mu}=\beta_{\mu}^{1}+i \beta_{\mu}^{2}$.

A formal calculation shows that the branch-lines are along the circles 


$$
\left\{\begin{array}{l}
x^{2}+\left(y-\beta_{\mu}^{2}\right)^{2}+\left(z+\beta_{\mu}^{1}\right)^{2}=\left(\beta_{\mu}^{1}\right)^{2}+\left(\beta_{\mu}^{2}\right)^{2} \\
y \beta_{\mu}^{1}+z \beta_{\mu}^{2}=0
\end{array}\right.
$$

which are tangent to the $x$-axis at the origin with centers at $\left(0, \beta_{\mu}^{2},-\beta_{\mu}^{1}\right)$. Since by hypotheses

$$
\beta_{\mu}=r\left(\cos \xi_{\mu}^{*}+i \sin \xi_{\mu}^{*}\right),
$$

the centers of the branch-line circles all lie on the circle (2.25).

Theorem 2.3. Let $\tilde{H}$ be defined by (2.21) and (2.22), with

$$
H-F^{* *}=\frac{1}{2 \pi i} \sum_{m=0}^{\infty} \sum_{k=-m}^{m-1} a_{m+k, m} \int_{|\zeta|=1} u^{m} \zeta^{k} \frac{d \zeta}{\zeta}
$$

and

$$
F^{* *}\left(X, Z, Z^{*}\right)=\frac{1}{2 \pi i} \sum_{n=0}^{\infty} a_{0, n} \int_{|\zeta|=1} u^{n} \zeta^{-n} \frac{d \zeta}{\zeta} .
$$

The hypothesis is the same as the previous theorem modified so that the distinguished sequence of coefficients $\left\{a_{0, n}\right\}$ is the subsequence along the left leg of (2.2).

The conclusion is the same as Theorem 2.2.

Proof. The proof is a modification of the proof of the previous theorem. Write (2.22) as

$$
\widetilde{H}=\frac{1}{2 \pi i} \int_{s}\left[\sum_{\mu=0}^{m} \frac{c_{\mu}}{u \zeta^{-1}-\gamma_{\mu}}+g^{*}\left(u \zeta^{-1}\right)\right] \frac{d \zeta}{\zeta},
$$

denoting the poles by

$$
\gamma_{1}, \cdots, \gamma_{p}
$$

For fixed $\mu$, integrating the first term gives

$$
c_{\mu} \int_{s} \frac{d \zeta}{\left(u \zeta^{-1}-\gamma_{\mu}\right) \zeta}=\frac{X+\sqrt{X^{2}-4 Z^{*}\left(Z-\gamma_{\mu}\right)}}{2\left(Z-\gamma_{\mu}\right) \sqrt{\bar{X}^{2}-4 Z^{*}\left(Z-\gamma_{\mu}\right)}}
$$

where the path $s$ is chosen appropriately (see remark after (2.28) and $\gamma_{\mu}=\gamma_{\mu}^{1}+i \gamma_{\mu}^{2}$.

A formal calculation shows that the branch-lines are along the circles

$$
\left\{\begin{array}{l}
x^{2}+\left(y-\gamma_{\mu}^{2}\right)^{2}+\left(z-\gamma_{\mu}^{1}\right)^{2}=\left(\gamma_{\mu}^{1}\right)^{2}+\left(\gamma_{\mu}^{2}\right)^{2} \\
y \gamma_{\mu}^{1}-z \gamma_{\mu}^{2}=0
\end{array}\right.
$$

A comparison shows that the circles (2.35) and (2.29) are in related families. 
Since the branch-lines depend only on the denominator of the integrand [4], Theorems 2.2 and 2.3 can be extended to summing over any parallel "diagonal" of (2.2). The branch-lines will be circles similarly situated, although the singular harmonic function will in general differ according to the diagonal distinguished. Indeed, for $n$ large enough the subseries associated with any "diagonal" parallel to the right leg can be written as

$$
u^{\nu}\left(\sum_{n} a_{2 n-\nu, n}(u \zeta)^{n}\right)
$$

3. Other rearrangements. It is possible to sum the series (2.1) by other rearrangements. Polar singularities associated with the distinguished subsequence of coefficients correspond to other possible singularity curves. In general, a distinguished subseries would be

$$
\frac{1}{2 \pi i} \sum_{n=0}^{\infty} \int_{|\zeta|=1} A_{n} u^{\kappa}\left(u^{N} \zeta^{M}\right)^{n} \zeta^{\omega} \frac{d \zeta}{\zeta}
$$

where $N, M, \kappa, \omega$ are fixed constants and $|M|<N$.

Interchanging summation and integration and supposing that the coefficients are such that the series has polar singularities similar to those of the previous theorems, the harmonic function (3.1) can be written

$$
\frac{1}{2 \pi i} \int_{|\zeta|=1} \frac{Q(u, \zeta) d \zeta}{a\left(u^{N} \zeta^{M}\right)^{m}+b\left(u^{N} \zeta^{M}\right)^{m-1}+\cdots}+\cdots
$$

where $Q(u, \zeta)$ is a polynomial in $u$ and $\zeta$.

The possible singularity curves of (3.2) depend only on the denominator of the integrand. Assuming that the polar singularities are simple the general class of possible singularity curves for (3.2) will be the same as for

$$
F\left(X, Z, Z^{*}\right)=\frac{1}{2 \pi i} \int_{|\zeta|=1} \frac{d \zeta}{\left(u^{N} \zeta^{M}-a\right) \zeta}
$$

where $\alpha$ is a complex number [14].

If $N \geqq 2$ the explicit evaluation of the integral (3.3) in closed form is difficult. Some information about the possible singularity curves, can be found by indirect means, however. It will be shown in the next theorem that the harmonic function (3.3) also satisfies a system of ordinary linear differential equations with rational coefficients. The theory of these ordinary differential equations can then be used to study the singularities of this class of solutions. 
(3.3) $\quad F\left(X, Z, Z^{*}\right)=\frac{1}{2 \pi i} \int_{|\zeta|=1} \frac{d \zeta}{\left(u^{N} \zeta^{M}-\alpha\right) \zeta}, \quad|N| \geqq M>0$

where $u=X+Z \zeta+Z^{*} \zeta^{-1}$, satisfies ordinary, linear, homogeneous differential equations, with respect to $X, Z, Z^{*}$, respectively, while keeping the remaining two variables fixed, of order

$$
r \leqq 2 N+1 \text {. }
$$

Proof: The proof will be given for the ordinary differential equation with respect to $X$ (keeping $Z, Z^{*}$ fixed). The proofs for the other cases are analogous.

For convenience, set

$$
\zeta=e^{i t}, d \zeta=i \zeta d t
$$

and write (3.3) as

$$
\begin{aligned}
F\left(X, Z, Z^{*}\right) & =\frac{1}{2 \pi i} \int_{0}^{2 \pi} \frac{e^{(N-M) i t} d t}{\left(Z e^{2 i t}+X e^{i t}+Z^{*}\right)^{N}-\alpha e^{(N-M) i t}} \\
& =\frac{1}{2 \pi i} \int_{0}^{2 \pi} V d t
\end{aligned}
$$

where

$$
V=\frac{e^{(N-M) i t}}{Q}
$$

and

$$
Q=\left(u e^{i t}\right)^{N}-\alpha e^{(N-M) i t} .
$$

The ordinary differential equation is of the form

$$
M(F) \equiv A_{0} F+A_{1} F_{x}^{1}+\cdots+A_{r} F_{x}^{[r]}=0 .
$$

Since

$$
F=\frac{1}{2 \pi i} \int_{0}^{2 \pi} V d t
$$

it is sufficient to prove that

$$
M(V) \equiv A_{0} V+A_{1} V_{x}^{1}+\cdots+A_{r} V_{x}^{[r]}=R(t)
$$

where

$$
R(t)=\frac{d}{d t}\left\{\left(1-e^{i t}\right) P V Q^{(1-r)}\right\}
$$

and 


$$
P=1+\sum_{\nu=1}^{l} p_{\nu} e^{i \nu t}
$$

the $p_{\nu}$ and $l$ to be determined.

The derivatives of $V$ with respect to $t$ and with respect to $X$ each have $V$ as a factor.

$$
\begin{aligned}
\frac{\partial V}{\partial t}= & V_{t}=V\left[i(N-M)-Q_{t} Q^{-1}\right] ; \\
\frac{\partial V}{\partial x}= & V_{x}=-V\left[N e^{i t}\left(u e^{i t}\right)^{N-1} Q^{-1}\right] ; \\
V_{x x}= & 2 V\left[e^{i t} N\left(u e^{i t}\right)^{N-1} Q^{-1}\right]^{2} \\
& -V\left[e^{2 i t} N(N-1)\left(u e^{i t}\right)^{N-2} Q^{-1}\right] ; \\
V_{x x x}= & -6 V\left[e^{i t} N\left(u e^{i t}\right)^{N-1} Q^{-1}\right]^{3} \\
& +6 V\left[e^{3 i t} N^{2}(N-1)\left(u e^{i t}\right)^{2 N-3} Q^{-2}\right] \\
& -V\left[e^{3 i t} N(N-1)(N-2)\left(u e^{i t}\right)^{N-3} Q^{-1}\right] .
\end{aligned}
$$

In general,

$$
V_{x}^{[r]}=\text { constant } \cdot V\left[e^{i t} N\left(u e^{i t}\right)^{N-1} Q^{-1}\right]^{r}+\cdots
$$

Expanding (3.6), obtain

$$
\begin{aligned}
R(t)=V Q^{-r}\left\{-i e^{i t}(N-M+1) P Q\right. \\
\left.+\left(i-e^{i t}\right)\left(P_{t} Q-r P Q_{t}\right)+(N-M) P Q\right\} .
\end{aligned}
$$

After substituting (3.9)-(3.13) into (3.5) multiply both sides of (3.5) by $Q^{r}$ and omit the common factor $V$. Then each side of the resulting equation is a polynomial in $\left(e^{i t}\right)$. If in (3.7), we choose

$$
l=2 N(r-1)-1
$$

then each side of the resulting equation will have the same degree, namely $2 N r$. Since equality holds for arbitrary $t$, the coefficients of each power of $\left(e^{i t}\right)$ on the left side may be equated to the coefficient of the same power on the right side of the equation.

Thus we obtain a system of $2 N r+1$ linear equations. If we choose

$$
r=2 N+1
$$

then the number of equations equals the number of unknown coefficients:

$$
A_{0}, A_{1}, \cdots, A_{r} ; p_{1}, p_{2}, \cdots, p_{l} ;\left(p_{0}=1\right) \text {. }
$$

In order to be able to determine these coefficients it suffices that the determinant $D\left(X, Z, Z^{*}\right)$ of these coefficients does not vanish identically. If $D \equiv 0$, it can easily be shown that there is a subdeterminant 
which does not vanish identically. In the case $D \equiv 0$, the order $r$ of the differential equation is less than $2 N+1$.

In that case where $D \equiv 0$, but the rank of $D$ is positive, we may use the linear dependence between the columns to determine the coefficients of the differential equation.

The rank of $D$ cannot be less than $l$ (see below). For the subdeterminant consisting of the columns corresponding to the coefficients $p_{1}, p_{2}, \cdots, p_{l}$, and the rows corresponding to $e^{i t}, e^{2 i t \cdots}, e^{l i t}$ is in triangular form with $\left(Z^{*}\right)^{N}$ on the main diagonal and zeros above the main diagonal. Assume that the rank $R \geqq l$ and that the nonvanishing $R$-rowed determinant is, for convenience, in the upper left hand corner.

If we denote the coefficients of the determinant by $\left\{c_{i, j}\right\}$, then because of the linear dependence of $R+1$ columns we can write

$$
c_{\mu, \delta}=\gamma_{1} c_{\mu 1}+\gamma_{2} c_{\mu_{2}}+\cdots+\gamma_{R} c_{\mu_{R}}
$$

where $\mu=1,2, \cdots, R ; \delta>R$.

From this system we can solve for $\gamma_{1}, \gamma_{2}, \cdots, \gamma_{R}$ and let

$$
\gamma_{1}=p_{1}, \gamma_{2}=p_{2}, \cdots, \gamma_{l}=p_{l} ; \gamma_{l+1}=A_{1}, \gamma_{l+2}=A_{2}, \cdots, \gamma_{R}=A_{R-l} \text {. }
$$

Similar theorems for certain classes of partial differential equations in two real variables have been proven. Bergman [1] showed that a class of solutions of $\Delta u+u(x, y)=0$ also satisfy ordinary linear differential equations. Nielsen [13] and Kreyszig [9], [10] have dealt with

$$
\Delta \psi+a(x, y) \frac{\partial \psi}{\partial x}+b(x, y) \frac{\partial \psi}{\partial y}+g(x, y) \psi=0
$$

4. A special case. A special, but typical, case of (3.3) will now be considered.

In (3.1) let $N=2, M=1, \kappa=0, \omega=0$. Then

$$
\left\{A_{n}\right\}=\left\{a_{3 n, 2 n}\right\}
$$

where the sequence of coefficients on the right refers to the triangular array (2.2). Specializing (3.3) gives

$$
F\left(X, Z, Z^{*}\right)=\frac{1}{2 \pi i} \int_{|\zeta|=1} \frac{d \zeta}{\left(u^{2} \zeta-\alpha\right) \zeta}
$$

The harmonic function (3.3)' also satisfies ordinary differential equations of order $r \leqq 5$. The differential equation with respect to $X$ will be constructed.

Denote 


$$
V=\frac{1}{u^{2} \zeta-\alpha}=\frac{e^{i t}}{\left(u e^{i t}\right)^{2}-\alpha e^{i t}}=\frac{e^{i t}}{Q}
$$

where $Q=\left(Z e^{2 i t}+X e^{i t}+Z^{*}\right)^{2}-\alpha e^{i t}$.

The differential equation to be constructed is

$$
M(V)=A_{0} V+A_{1} V_{x}^{1}+\cdots+A_{5} V_{x}^{[5]}=R(t)
$$

where

$$
\begin{aligned}
R(t) & =\frac{d}{d t}\left\{\left(1-e^{i t}\right) V P Q^{-4}\right\} \\
& =V Q^{-5}\left\{-2 i e^{i t} P Q+\left(i-e^{i t}\right)\left(P_{t} Q-5 P Q_{t}\right)+P Q\right\}
\end{aligned}
$$

By formal computation

$$
\begin{aligned}
V & =e^{i t} Q^{-1} ; \\
V_{x} & =-2 V\left\{V\left(u e^{i t}\right)\right\} ; \\
V_{x}^{\prime \prime} & =V\left\{-2 e^{i t} V+8\left(u e^{i t}\right)^{2} V^{2}\right\} ; \\
V_{x}^{\prime \prime \prime} & =V\left\{24\left(u e^{i t}\right) e^{i t} V^{2}-48\left(u e^{i t}\right)^{3} V^{3}\right\} ; \\
V_{x}^{[4]} & =V\left\{24 e^{2 i t} V^{2}-288\left(u e^{i t}\right)^{2} e^{i t} V^{3}+344\left(u e^{i t}\right)^{4} V^{4}\right\} ; \\
V_{x}^{[5]} & =V\left\{-720\left(u e^{i t}\right) e^{2 i t} V^{3}+3680\left(u e^{i t}\right)^{3} e^{i t} V^{4}-3440\left(u e^{i t}\right)^{5} V^{5}\right\} .
\end{aligned}
$$

Substituting (4.3) and (4.4) into (4.2), multiplying both sides by $Q^{5}$ and omitting the common factor $V$, we obtain an equation of polynomials each of degree 20 in $\left(e^{i t}\right)$ from which is obtained a linear system of twenty-one equations in the twenty-one unknowns

$$
A_{0}, A_{1}, \cdots, A_{5} ; p_{1}, \cdots, p_{15} \text {, }
$$

which can be solved. $p_{0}=1$.

Equating the coefficients of the same powers of $\left(e^{i t}\right)$ we have

$$
\begin{aligned}
& \left(e^{i t}\right)^{0}: A_{0}\left(Z^{* 10}\right)=i Z^{* 2} \\
& \left(e^{i t}\right): A_{0}\left(10 X Z^{* 9}-5 Z^{* 8} \alpha\right)+A_{1}\left(-2 Z^{* 9}\right) \\
& =i\left(-2 Z^{* 2}-8 X Z^{*}-4 \alpha\right)+i p_{1}\left(2 Z^{* 2}\right) \\
& \left(e^{i t}\right)^{19}: A_{0}\left(10 X Z^{9}\right)+A_{1}\left(-2 Z^{9}\right)=i p_{15}\left(-28 Z X-4 Z^{2}\right)+i p_{14}\left(-12 Z^{2}\right) \\
& \left(e^{i t}\right)^{20}: A_{0}\left(Z^{10}\right)=i p_{15}\left(-13 Z^{2}\right) \text {. }
\end{aligned}
$$

It can immediately be seen that the coefficient

$$
A_{0}=i\left(Z^{*}\right)^{-8} \text {. }
$$

Integration with respect to $t$ from 0 to $2 \pi$ will not affect the coefficients but (4.2) will become

$$
M(F)=0
$$


If the linear system is arranged so that the $k$ th row corresponds to $\left(e^{i t}\right)^{k-1}$ and the columns from left to right correspond to $p_{1}, p_{2}, \cdots, p_{15}$, $A_{0}, \cdots, A_{5}$ the determinant of the coefficients on the left side of the system has an interesting form.

The elements of the first row are zero except for the column corresponding to $A_{0}$, which element is $Z^{* 10}$. Denoting elements of $D$ by $\left(c_{p q}\right)$ we have

$$
c_{\nu, \nu-1}=i \nu Z^{* 2}, \quad \nu=2,3, \cdots, 16 .
$$

while the elements in the rows above these elements are zero. We also have the elements

$$
c_{7,1}, c_{8,2}, c_{9,3}, \cdots, c_{21,15}
$$

each equal to $\left(Z^{2}\right)$ multiplied by an imaginary constant, while the elements in the rows below (4.8) are all zero.

The $6 \times 6$ matrix in the upper right hand corner is in triagnular form where the elements of the main diagonal are: const. $Z^{* 10}$, const. $Z^{* 9}, \cdots$, const. $Z^{* 5}$.

The $6 \times 6$ matrix in the lower right hand corner has only zero elements below the other diagonal and on that diagonal, the elements are, (reading from bottom to top and left to right).

$$
\text { const. } Z^{10} \text {, const. } Z^{9}, \cdots \text {, const. } Z^{5} \text {. }
$$

The possible singular curves of the solutions of the differential equation will be those curves for which the determinant $D$ is zero. It is easy to see that the columns are linearly independent and hence that the determinant does not vanish identically.

These results on singularities can be extended to the equation

$$
\frac{\partial^{2} \phi}{\partial x^{2}}+\frac{\partial^{2} \phi}{\partial y^{2}}+\frac{\partial^{2} \phi}{\partial z^{2}}+f\left(r^{2}\right) \phi=\frac{\partial^{2} \tilde{\phi}}{\partial x^{2}}-\frac{\partial^{2} \tilde{\phi}}{\partial Z \partial Z^{*}}+f \tilde{\phi}=0
$$

where $Z=\frac{1}{2}(z+i y), Z^{*}=-\frac{1}{2}(z-i y), r^{2}=x^{2}+y^{2}+z^{2}=x^{2}-4 Z Z^{*}$. See [2], [3].

\section{REFERENCES}

1. S. Bergman, Üeber Kurvenintegrale von Funktionen zweier komplexer Veraenderlicher, die Differentialgleichung $\Delta V+V=0$ befriedigen, Math. Z., 32 (1930), 386-405.

2. - On solutions with algebraic character of linear partial differential equations, Trans. Amer. Math. Soc., 68 (1950), 461-507.

3. - Essential singularities of a class of linear partial differential equations in three variables. J. Rat. Mech. Analysis 3 (1954), 539-560.

4. - Integral Operators in the Theory of Linear Partial Differential Fquations, Ergebnisse der Mathematik, N. F. 23 (1961).

5. - Some properties of a harmonic function of three variables given by its series 
development, Arch. for Rat. Mech. and Analysis 8 (1961), 207-222.

6. P. Dienes, The Taylor Series, Dover Edition; New York, (1957).

7. R. P. Gilbert, Singularities of three-dimensional harmonic functions, Pacific J. Math., 10 (1960), 1243-1255.

8. Hadamard et Mandelbrojt, La Série de Taylor et son prolongement analytique, GauthierVillars, Paris, (1926).

9. E. Kreyszig, On certain partial differential equations and their singularities, J. Rat. Mech. Analysis, 5 (1956), 805-820.

10. On some relations between partial and ordinary differential equations, Can. J. Math., 10 (1958), 183-190.

11. S. Mandelbrojt, Théoréme général fournissant l'argument des points singuliers situés sur le cercle de convergence de la séries de Taylor, C. R. Acad. Science, Paris, 204 (1937), 1456-1458.

12. J. Mitchell, Some properties of solutions of partial differential equations given by their series development, Duke Math. Journ., 13 (1946), 87-104.

13. K. J. Nielsen, Some properties of functions satisfying partial differential equations of elliptic type, Duke Math. Journ., 11 (1944), 121-137.

14. A. White, Singularities of harmonic functions of three variables generated by Whittaker-Bergman operators, Annales Polonici Math., 10 (1961), 81-100.

15. Whittaker, Watson, A Course of Modern Analysis, third ed., Cambridge, (1920). 



\section{PACIFIC JOURNAL OF MATHEMATICS}

\section{EDITORS}

\section{RalPh S. Phillips}

Stanford University

Stanford, California

M. G. Arsove

University of Washington

Seattle 5, Washington
J. Dugundji

University of Southern California Los Angeles 7, California

Lowell J. Paige

University of California

Los Angeles 24, California

\section{ASSOCIATE EDITORS}
E. F. BECKENBACH
D. DERRY
H. L. ROYDEN
E. G. STRAUS
T. M. CHERRY
M. OHTSUKA
E. SPANIER
F. WOLF

\section{SUPPORTING INSTITUTIONS}

\author{
UNIVERSITY OF BRITISH COLUMBIA \\ CALIFORNIA INSTITUTE OF TECHNOLOGY \\ UNIVERSITY OF CALIFORNIA \\ MONTANA STATE UNIVERSITY \\ UNIVERSITY OF NEVADA \\ NEW MEXICO STATE UNIVERSITY \\ OREGON STATE UNIVERSITY \\ UNIVERSITY OF OREGON \\ OSAKA UNIVERSITY \\ UNIVERSITY OF SOUTHERN CALIFORNIA
}

STANFORD UNIVERSITY

UNIVERSITY OF TOKYO

UNIVERSITY OF UTAH

WASHINGTON STATE UNIVERSITY

UNIVERSITY OF WASHINGTON

AMERICAN MATHEMATICAL SOCIETY

CALIFORNIA RESEARCH CORPORATION SPACE TECHNOLOGY LABORATORIES

NAVAL ORDNANCE TEST STATION

Printed in Japan by International Academic Printing Co., Ltd., Tokyo Japan 


\section{Pacific Journal of Mathematics}

\section{Vol. 13, No. 1 \\ March, 1963}

Frantz Woodrow Ashley, Jr., A cone of super-(L) functions............. 1

Earl Robert Berkson, Some metrics on the subspaces of a Banach space....

Felix Earl Browder and Walter Strauss, Scattering for non-linear wave

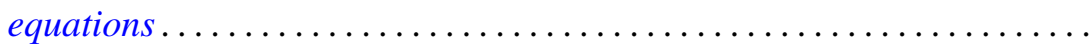

Edmond Darrell Cashwell and C. J. Everett, Formal power series ..........

Frank Sydney Cater, Continuous linear functionals on certain topological

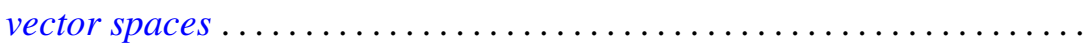

John Douglas Dixon, General group extensions ....................

Robert Pertsch Gilbert, On harmonic functions of four variables with

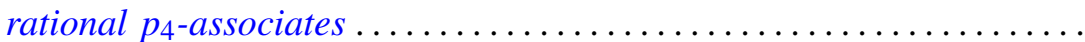

Irving Leonard Glicksberg, On convex hulls of translates ..............

Simon Hellerstein, On a class of meromorphic functions with deficient zeros

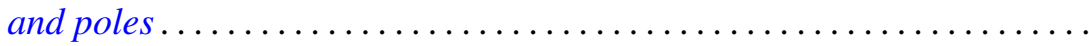

Donald William Kahn, Secondary cohomology operations which extend the

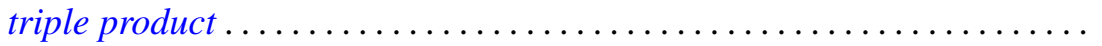

G. K. Leaf, A spectral theory for a class of linear operators .............

R. Sherman Lehman, Algebraic properties of the composition of solutions of partial differential equations ........................... 157

Joseph Lehner, On the generation of discontinuous groups ............. 169

S. P. Lloyd, On certain projections in spaces of continuous functions ...... 171 Fumi-Yuki Maeda, Generalized spectral operators on locally convex spaces ..................................

Donald Vern Meyer, $E^{3}$ modulo a 3-cell

William H. Mills, An application of linear programming to permutation groups.

Richard Scott Pierce, Centers of purity in abelian groups

Christian Pommerenke, On meromorphic starlike functions ...

Zalman Rubinstein, Analytic methods in the study of zeros of

polynomials...

B. N. Sahney, On the Nörlund summability of Fourier series

Tôru Saitô, Regular elements in an ordered semigroup . .

Lee Meyers Sonneborn, Level sets on spheres...........

Charles Andrew Swanson, Asymptotic estimates for limit point

problems .

Lucien Waelbroeck, On the analytic spectrum of Arens . .

Alvin (Murray) White, Singularities of a harmonic function of three

variables given by its series development .............

Kōichi Yamamoto, Decomposition fields of difference sets ...

Chung-Tao Yang, On the action of $\mathrm{SO}(3)$ on a cohomology manifold... 